\title{
Implications of Spatial Scales and Reporting Units for the Spatial Modelling of Vulnerability to Vector-borne Diseases
}

\author{
Michael HAGENLOCHER, Stefan KIENBERGER, \\ Stefan LANG and Thomas BLASCHKE \\ Interfaculty Department of Geoinformatics - Z GIS, \\ University of Salzburg / Austria·michael.hagenlocher@sbg.ac.at
}

This contribution was double-blind reviewed as full paper.

\begin{abstract}
Vector-borne diseases (VBDs) impose a heavy burden on vulnerable populations. Although vulnerability has increasingly gained ground as a promising concept in VBD literature in recent years, little emphasis has been placed on developing methods for quantifying and mapping it. Integrated spatial indicators are a powerful means for grasping the complexity of vulnerability in a spatially explicit manner, and translating it into policy-relevant information. Based on the 'vulnerability cube' concept, this paper presents lessons learnt from a benchmarking exercise of three spatial vulnerability assessments. The assessments were carried out for two different diseases, malaria and dengue fever, at three different spatial scales (local, national, and regional), using three different spatial reporting units (administrative units, pixels, and geons). We found that the choice of the spatial scale and the reporting unit for modelling, visualizing, and communicating vulnerability to VBDs has both conceptual and methodological/practical implications that developers and users of vulnerability indices should be aware of.
\end{abstract}

\section{Introduction}

\subsection{The global burden of malaria and dengue fever}

Vector-borne diseases (VBDs) are infectious diseases caused by pathogens transmitted among hosts by intermediate species (MAGORI \& DRAKE 2013). The two target diseases of this study, malaria and dengue fever, are transmitted by mosquitoes, albeit by different species. Globally, malaria is the most prevalent VBD. Caused by an infection with protozoa of the genus Plasmodium, transmitted through the bite of the Anopheles mosquito, malaria resulted in approximately 207 million infections and 627.000 deaths in 2012, mostly among African children under five years of age (WHO 2013). Dengue fever, a viral infection transmitted by the bite of the Aedes aegypti mosquito, is currently the world's fastest growing VBD (MAGORI \& DRAKE 2013). Its incidence has increased 30-fold over the past 50 years. It primarily affects urban and sub-urban environments of the sub-/tropics, and causes an estimated number of 50-100 million infections every year (SIMMONS et al. 2012). Despite recent progress in prevention and control, malaria and dengue fever still make a 
major contribution to the global infectious disease burden today, which is quantified in terms of disability-adjusted life years (DALYs).

\subsection{Concept of risk and vulnerability to VBDs}

Today, most prevention and control strategies for mosquito-borne diseases tend to concentrate on (1) reducing exposure to the mosquito through a combination of indoor residual spraying (IRS), and the promotion and distribution of long-lasting insecticidal nets (LLINs), or (2) treating infections, for example through administering antimalarial drugs, etc. While these strategies provide tangible health benefits, the high burden of these two diseases calls for novel ways to further reduce risk among the most vulnerable: namely from an integrative perspective that connects the environmental (incl. climatic), social, demographic, economic, biological, cultural and political factors which altogether contribute to risk (STRATTON et al. 2008).

In the context of VBDs, risk is a function of the hazard, i.e. disease prevalence (or the likelihood of an infective bite), and the vulnerability of exposed population groups (Fig. 1). The interrelation of these three components reflects the vital importance of feedback when dealing with a human-vector transmission cycle. As a primary focus on hazard interventions has not resulted in the desired reduction of risk, vulnerability has been established as a key concept within the disaster risk reduction and climate change adaptation

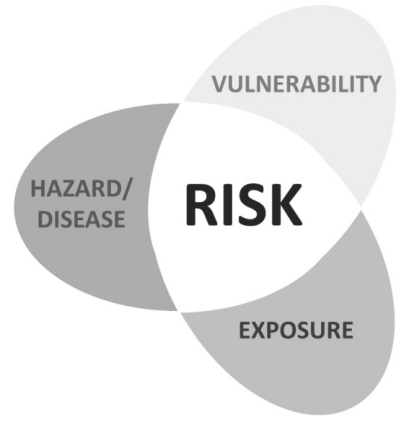

Fig. 1: Risk framework communities over the past decades (IPCC 2014). Due to its promise for public health, the focus on vulnerability to VBDs has increasingly gained ground in scientific literature in recent years. However, little emphasis has been placed on developing methods for assessing it in a spatially explicit manner. Vulnerability is defined as the predisposition of the population to the burden of the disease. It is characterized by different interrelated domains, (1) generic and biological susceptibility (SUS), and (2) lack of resilience (LoR), and takes into account different dimensions (e.g., social, economic, etc.) and factors that lead to differences in the impact of the disease. The distinction between generic and biological factors pre-disposing a community to disease burden, such as poverty, nutritional status, lacking immunity, etc., versus those that determine its ability to anticipate (use of LLINs, IRS, housing conditions, education, etc.), respond to/cope with (access to health care, etc.), and recover from that burden should be relevant for decision makers. It helps prioritizing targeted interventions that aim at reducing prevailing susceptibilities and strengthening resilience to VBDs.

\subsection{Aim of this study}

Integrated spatial indicators (also referred to as 'spatial composite indicators') provide a powerful means for grasping the complexity of multi-dimensional vulnerability to VBDs in a spatially explicit manner. Next to indicator choice, spatial scale and the selection of an 'appropriate' reporting unit (in the sense of LANG et al. 2010) are important, yet often neglected elements for all vulnerability assessments. This paper targets two of these elements by identifying conceptual and methodological/practical implications of (1) spatial scale, and (2) the choice of the reporting unit for spatial vulnerability assessments. 
Currently, and to our best knowledge, no study exists that reviews the implications of both spatial scales and reporting units. To close this gap we benchmarked three case studies that were carried out at different spatial scales (local, national, and regional), using different reporting units (administrative units, continuous grids, and geons).

\section{Methodology}

\subsection{Case studies}

Based on the conceptual risk and vulnerability framework, we have modeled relative levels of vulnerability to VBDs in three different study areas (Table 1; Figure 2). Thereby, we followed a workflow that comprises the following modeling stages: indicator choice and data pre-processing - including the detection and treatment of missing data and outliers, as well as an assessment of existing multicollinearities in the data, normalization, weighting, aggregation, and visualization (HAGENLOCHER et al. 2013, LANG et al. 2014).

Tab. 1: Overview of the three case studies, including information on the target disease, spatial scale and the choice of the reporting unit for modelling, visualizing and communicating relative levels of vulnerability

\begin{tabular}{|c|l|c|c|c|}
\hline \multicolumn{2}{|c|}{} & \multicolumn{3}{|c|}{ Reporting unit/s } \\
\cline { 3 - 5 } & Administrative units & Grids/pixels & Geons \\
\hline \multirow{2}{*}{$\begin{array}{c}\# 1-\text { Cali, Colombia } \\
\text { (dengue fever) }\end{array}$} & Local & $\begin{array}{c}\# 2-\text { Tanzania } \\
\text { (malaria) }\end{array}$ & $\begin{array}{c}\# 2-\text { Tanzania } \\
\text { (malaria) }\end{array}$ & N/A \\
\cline { 2 - 5 } & National & N/A & N/A & $\begin{array}{c}\# 3-\text { Eastern Africa } \\
\text { (malaria) }\end{array}$ \\
\cline { 2 - 5 } & Regional & & & \\
\hline
\end{tabular}

The first study (\#1) was carried out in Cali, Colombia. With an estimated population of 2.3 million Cali is currently the country's third largest city. Based on a set of 17 socioeconomic and demographic indicators derived from census data and ancillary geospatial datasets, we modelled socioeconomic vulnerability to dengue fever on a local scale. The single indicators were assigned to their respective vulnerability domains (SUS, LoR), before aggregating the two domains into a composite index of socioeconomic vulnerability. Weights were identified using expert opinions $(n=4$ local domain experts) and principal component analysis, revealing high concordance between both approaches. As the indicators were reported at neighbourhood level, the resulting composite vulnerability index was also constructed and visualized using administrative units. A detailed description of the study is provided by HAGENLOCHER et al. (2013), while the visualization of the vulnerability index in an interactive WebGIS environment is discussed by KIENBERGER et al. (2013a).

In the second case study (\#2) we modelled malaria vulnerability in Tanzania using a set of 20 socioeconomic, demographic, biological, environmental, and governance-related indicators. The single indicators were aggregated into their respective domains (SUS, LoR) 
using equal weights, which were then, in a next step, combined into a composite vulnerability index. The analysis was carried out on a national scale for the entire country using spatially disaggregated datasets. Results were visualized using both $10 \times 10 \mathrm{~km}^{2}$ grids, and sub-national administrative units. Details of this study, including information on the vulnerability indicators, will be published later this year.

The third case study (\#3) covers the entire East African Community (EAC) region, comprising Burundi, Kenya, Rwanda, Tanzania and Uganda. Here, we modelled social vulnerability to malaria on a regional scale. In contrast to the previous case studies, indicators were not aggregated by vulnerability domains, but by thematic dimension. The 15 indicators were resampled to continuous datasets of $10 \times 10 \mathrm{~km}^{2}$ spatial resolution, before integrating them in an index. Weights for the single indicators were identified using expert opinions ( $n=7$ regional domain experts). We delineated homogeneous 'vulnerability units', so-called integrated geons (LANG et al. 2014) using a regionalization approach (HAGENLOCHER et al. 2014). While operational in object-based image analysis, utilizing segmentation algorithms for delineating 'objects' representing latent phenomena is a novel and innovative way of creating homogeneous units that are independent of a-priori defined artificial boundaries (e.g., administrative units).

\subsection{The 'vulnerability cube'}

As indicated earlier, we used an adapted version of the 'vulnerability cube' for benchmarking the three case studies. The vulnerability cube was initially introduced by LIN \& MOREFIELD (2011), who used it to categorize vulnerability index values within a threedimensional variable space. Our study builds on a recent version of the vulnerability cube published by KIENBERGER et al. (2013b), who used it as a basis for comparing existing vulnerability assessments by positioning them in a three-dimensional space; where each dimension represents one axis of the cube. The framework proposed by KIENBERGER et al. (2013b) used space (WHERE), time (WHEN), and thematic dimension (WHAT) for the identification of overlaps, differences, and specific characteristics of 20 vulnerability assessments in a natural hazard and/or climate change context.

In our study we modified the axes to fit our particular needs, as well as the characteristics of the three case studies described above. As this paper aims at comparing assessments at different spatial scales, we assigned 'space' (here: spatial scale) to the x-axis. We also kept the 'dimensions' (here: vulnerability dimensions) on the z-axis. However, as all case studies were a snapshot in time, we replaced 'time' with 'reporting unit' on the y-axis (Figure 3). This was done to identify benefits and challenges of using different reporting units, thus replacing the 'WHEN' by 'HOW'.

\section{Results and Discussion}

\subsection{Modelling outputs and adapted vulnerability cube}

Figure 2 shows the modelling outputs of the three case studies: socioeconomic vulnerability to dengue fever in Cali, Colombia (left), malaria vulnerability in Tanzania (middle), and social vulnerability to malaria for the EAC region (right). Areas of high vulnerability are 
displayed in shades of red, while those of low vulnerability are visualized in shades of blue. For Tanzania, the results were visualized both using $10 \times 10 \mathrm{~km}^{2}$ grids, and sub-national administrative units.
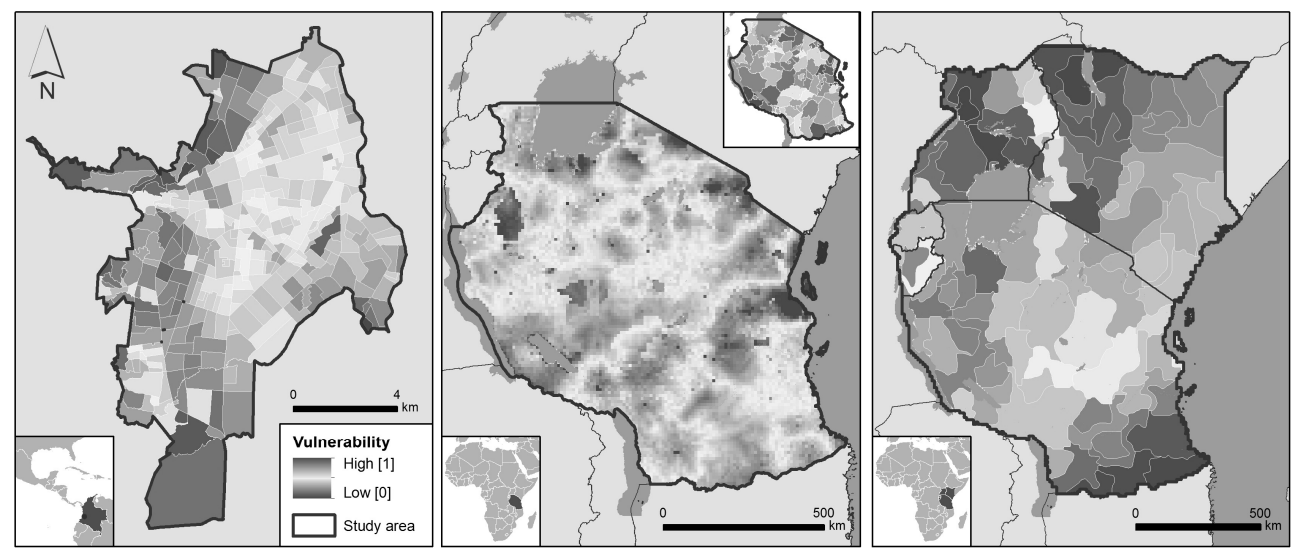

Fig. 2: Outcomes of the spatial vulnerability assessments in Cali, Colombia (left), the United Republic of Tanzania (middle), and eastern Africa (right)

Figure 3 shows the adapted vulnerability cube that was used for the identification of overlaps and differences between the three case studies in regards to spatial scale, vulnerability dimension(s), and the choice of the final reporting unit.

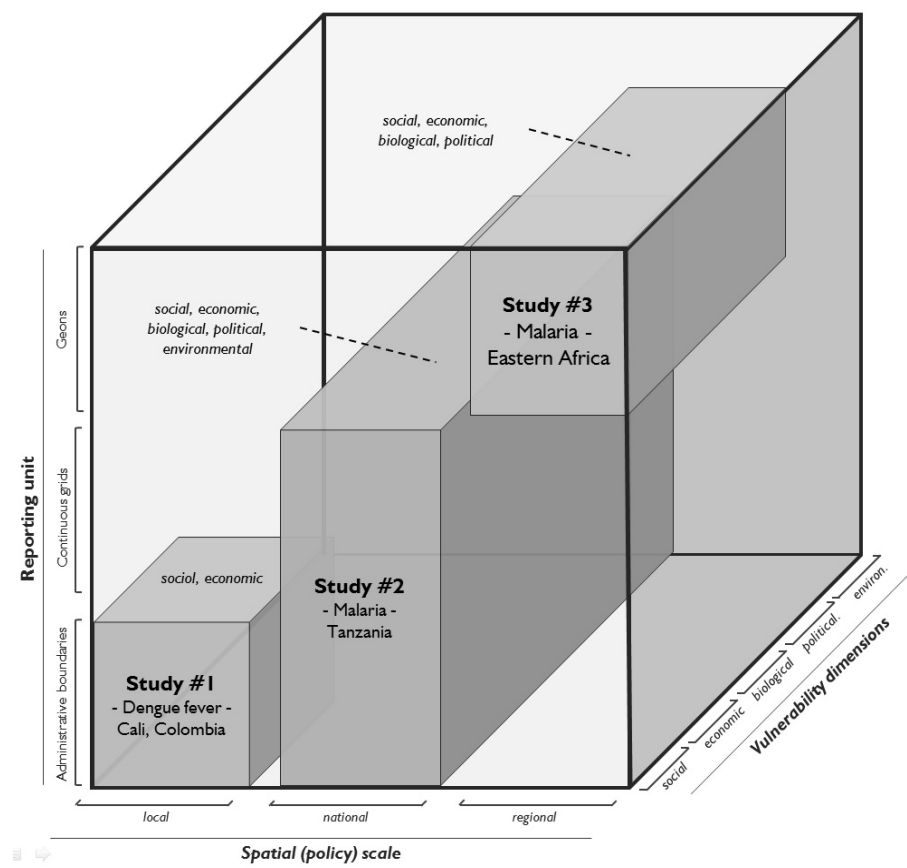

Fig. 3: Vulnerability cube for benchmarking the three case studies 
The cube helps to grasp both differences and overlaps between the case studies in an appealing and structured way. Thus it provides a valuable alternative to simple comparisons in a table. Based on the interpretation of the vulnerability cube, and the experiences drawn from the three case studies, the following paragraphs discuss the implications of (1) spatial scale, and (2) the choice of the final reporting unit for spatial vulnerability assessments.

\subsection{Implications of spatial scale}

Conducting spatial vulnerability assessments at different spatial scales has implications both on a conceptual and a methodological/practical level that developers should be aware of. A detailed introduction on spatial scales as a challenge for spatial vulnerability assessments in a natural hazard context has been published by FEKETE et al. (2010) and KIENBERGER et al. (2013a), who, among other things, highlighted that more attention should be devoted to scale implications. So far, quantitative vulnerability studies mainly focussed on the analysis of existing spatial data, while assuming that this data would reflect the appropriate spatial pattern in some profound sense. BLASCHKE (2006) argues that spatial pattern fundamentally matters, because context can have a fundamental influence of meaning and value: different structures have different implications for processes. By implication, therefore, vulnerability studies should consider spatial patterns as an issue in its own right. FEKETE et al. (2010) emphasize that vulnerability is nested at different spatial scales, interwoven in complex ways, thus calling not only for assessments at different levels (multi-scale), but also for modelling vulnerability across scales (cross-scale).

An overview of challenges arising from cross-scale analysis is provided by FEKETE et al. (2010). The focus of this study is placed on multi-scale implications for spatial vulnerability assessments. Drawing on the experiences from the three case studies that were carried out at different spatial/policy scales (local, national, regional), table 2 lists different aspects (e.g., data availability, etc.) and related challenges for spatial vulnerability assessments at different scales. According to WU \& HARBIN (2006) it is important to match the modelling scale with the policy scale; i.e. the scale at which decisions are made. As vulnerability assessments aim at supporting decision making processes, we use spatial scale synonymously to policy scale.

Tab. 2: Implications of spatial scales for spatial vulnerability assessments: while some aspects barely pose a challenge $(\bullet)$ for assessments at one particular scale, they pose a medium $(\bullet \bullet)$, or even major challenge $(\bullet \bullet \bullet)$ at other scales.

\begin{tabular}{|l|c|c|c|}
\hline & Local scale & National scale & Regional scale \\
\hline Data availability & $\bullet$ & $\bullet \bullet$ & $\bullet \bullet \bullet$ \\
\hline Data collection & $\bullet$ & $\bullet \bullet$ & $\bullet \bullet \bullet$ \\
\hline Uncertainty in the data & $\bullet$ & $\bullet \bullet$ & $\bullet \bullet \bullet$ \\
\hline Level of spatial detail & $\bullet$ & $\bullet \bullet$ & $\bullet \bullet \bullet$ \\
\hline $\begin{array}{l}\text { Identification of root causes of } \\
\text { vulnerability }\end{array}$ & $\bullet$ & $\bullet \bullet \bullet$ & $\bullet \bullet \bullet$ \\
\hline $\begin{array}{l}\text { Availability/reliability of } \\
\text { expert knowledge }\end{array}$ & $\bullet$ & $\bullet \bullet$ & $\bullet \bullet$ \\
\hline Validation & $\bullet$ & $\bullet$ & $\bullet \bullet$ \\
\hline
\end{tabular}


Comparing the three case studies, we found that data availability decreases with coarser spatial resolution, while uncertainty in the data also grows with increasing extent (e.g., as a result of data generalization, or the use of proxies as indirect surrogates on coarser spatial scales). Assessments at a regional scale, and particularly in data scarce environments, face the additional challenge that while relevant datasets might be available for one country, they might not for another. Within the assessment in eastern Africa (case study \#3) we were confronted with the situation that data availability is high for Tanzania and Kenya, acceptable for Rwanda and Burundi, yet extremely poor for Uganda. At the same time collecting a representative sample of data using surveys or participatory methods is much easier on a local scale than doing so on a national or even regional scale. For example, the study in Cali (study \#1) was carried out using census data provided by the local authorities, while the analyses in Tanzania (study \#2) and eastern Africa (study \#3) were largely based on existing geospatial datasets (e.g., gridded population datasets, etc.) that are available for the entire African continent or even globally. Therefore, the dependency on existing datasets also increases with increasing spatial extent. We also found that data on the local level is often reported on pre-defined 'artificial boundaries', such as census units, neighbourhoods or other administrative boundaries. Apart from the fact that this hampers assessing the 'real' spatial distribution of vulnerability to a given hazard/disease, this may also lead to aggregation problems as described by OPENSHAW (1984). As empirical models are largely not available, expert opinion is a plausible alternative to equal weighting when defining the relevance of the single indicators for the composite vulnerability index. Developers of spatial vulnerability indices, however, should be aware that the availability of domain experts with relevant "local knowledge" for judging the importance of the single indicators, identifying and exploring the root causes of vulnerability, or validating the final vulnerability maps, also decreases with increasing extent of the study area. We hypothesize that the subjectivity of expert judgement also increases with coarser spatial scale, likely resulting in a higher chance of biased judgements when assigning weights to indicators. For example, FEIZIZADEH et al. (2014) assessed the bias in expert judgements using the analytic hierarchy process (AHP). HAGENLOCHER et al. (2013), however, compared the impact of expert-based and statistical weighting approaches on a vulnerability index using local sensitivity analysis and found that both approaches revealed similar outputs, concluding that in the absence of local expertise, statistical approaches could be used. A detailed review of scale implications for such qualitative information is provided in KIENBERGER et al. (2013b). Finally, assessments at a local scale provide a very high level of spatial detail, revealing relevant information (e.g., detection of small-scale patterns) for local interventions. The level of detail also decreases with assessments at coarser scales, which is primarily a result of the resolution of available datasets (e.g., information on household level for local scales versus gridded datasets for assessments at national or regional scales). However, working on a coarser scale (national or regional) provides national or regional policy- and decision makers with the required overview, as it enables both a comparison between regions, as well as the identification of larger patterns and 'vulnerability hotspots', which require further in-depth analysis on a sub-national or local scale. Finally, we found that using a conceptual framework for the operationalization of vulnerability was of utmost importance when benchmarking the three case studies, as such a framework not only enables a transferability of the modelling approach within the same spatial scale, but it also serves as a 'guidance tool' for assessments at different spatial scales (multi- and crossscale). 


\subsection{Implications of the choice of the reporting unit}

Based on the comparative evaluation of the three study areas, table 3 provides an overview of the methodological implications of the choice of the spatial reporting unit for modelling vulnerability to VBDs in a spatially explicit manner. Three different types of units are considered: (1) administrative boundaries, (2) continuous grids, and (3) geons. The benchmarking of the three case studies has shown that the choice of the reporting unit also has relevant practical or methodological implications that developers of vulnerability indices should be aware of.

Tab. 3: Implications of spatial reporting units for spatial vulnerability assessments: while some aspects barely pose a challenge $(\bullet)$ for assessing vulnerability when using one particular reporting unit, they pose a medium $(\bullet \bullet)$, or even major challenge $(\bullet \bullet \bullet)$ when using another.

\begin{tabular}{|l|c|c|c|}
\hline & Admin. units & Grids/pixels & Geons \\
\hline Modifiable areal unit problem (MAUP) & $\bullet \bullet \bullet$ & $\bullet \bullet \bullet$ & $\bullet$ \\
\hline Internal heterogeneity/variance & $\bullet \bullet \bullet$ & $\bullet \bullet$ & $\bullet$ \\
\hline Spatial explicit representation of vulnerability & $\bullet \bullet \bullet$ & $\bullet$ & $\bullet$ \\
\hline Intrinsic geographical meaning of the unit & $\bullet \bullet \bullet$ & $\bullet \bullet \bullet$ & $\bullet$ \\
\hline $\begin{array}{l}\text { Size and shape of units change when } \\
\text { modelling/input parameters change }\end{array}$ & $\bullet$ & $\bullet$ & $\bullet \bullet \bullet$ \\
\hline Comprehensible for policy-/decision makers & $\bullet$ & $\bullet \bullet \bullet$ & $\bullet \bullet$ \\
\hline Matches scale of current interventions & $\bullet$ & $\bullet \bullet \bullet$ & $\bullet \bullet \bullet$ \\
\hline Useful for intervention planning & $\bullet \bullet$ & $\bullet \bullet \bullet$ & $\bullet$ \\
\hline
\end{tabular}

Until today, most spatial vulnerability assessments are carried out using administrative boundaries as the final reporting unit (KIENBERGER et al. 2013a, PRESTON et al. 2010). As most interventions in a VBD context (e.g., IRS, or the distribution of LLINs) are spatially targeted at the administrative level, results reported on administrative units match the scale of current policy interventions. Results reported on the administrative level are easily comprehensible by policy- and/or decision makers on the ground, as they have a long tradition using them. As administrative units rarely change over time, continuous monitoring of spatiotemporal changes of vulnerability can be easily achieved. This also holds true for the evaluation of the response of the composite vulnerability index to variations in single or multiple input parameters. Such a sensitivity analysis can be easily performed on administrative unit level, as the units remain constant. However, as pointed out by OPENSHAW (1984), the results and their interpretation have to be treated with caution as they are 'biased' by the fact that administrative units are modifiable, artificial areal units. Continuous grids, or pixels, enable spatially explicit modelling and monitoring, as well as a straightforward evaluation of the sensitivity of the modelling approach. However, they do not correspond to real world 'objects', and thus - like administrative boundaries - do not have an intrinsic geographical meaning. As discussed in OPENSHAW (1984), descriptive statistics change when resampling the grid cells. The geon approach (LANG et al. 2014), instead generates boundaries where the phenomenon under concern changes its overall behaviour. In contrast to administrative units and grids they provide a representation of the 
real spatial distribution of vulnerability to VBDs. Since several indicators are integrated, internal variations are retained. Although geons do not necessarily match the scale of current policy interventions, thus calling for a paradigm shift in intervention planning, they provide a powerful means for intervention planning as their internal heterogeneity/variance is minimized (ibid.). Thus, for geons vulnerability reduction measures do not need to be spatially targeted within reporting units; something that is rarely the case for administrative units. For these reasons, geons can be considered an ideal representation of the inherent spatial heterogeneity of vulnerability, whilst boundaries may be subject to change with altering vulnerability patterns. To create such on-demand geographies (LANG et al. 2014) means sacrificing fixed and independently defined boundaries for the sake of units that are characterised by a uniform behaviour of the vulnerabilities assessed.

\section{Conclusion and Outlook}

Depending on the choice of the spatial scale and the reporting unit for modelling and visualizing vulnerability, the developers of composite vulnerability indices are confronted with a range of conceptual and methodological implications they should be aware of. This paper presents a collection of both scale implications and implications of the choice of the reporting unit based on the experience drawn from three case studies. According to our benchmarking analysis, the choice of the scale of analysis has multiple implications on the development of composite vulnerability indicators. This includes: (1) data availability, (2) data collection, (3) data quality (uncertainty in the data), (4) the availability of domain experts for revealing the root causes of vulnerability, weighting indicators or validating the results (this is also related to the extent of the study area), (5) the level of spatial detail in the results, as well as issues around (6) whether the scale of analysis matches with the scale of interventions. The benchmarking exercise has also shown that, depending on the choice of the reporting unit, the following issues have to be considered: (1) MAUP, (2) systemic stability of the units (referring to their internal heterogeneity), (3) whether or not the units have an intrinsic geographical meaning or allow for spatially explicit modelling, (4) linkages between current intervention planning approaches and the usefulness of the chosen unit for intervention planning. While care must be taken whatever combination of scale and reporting unit is applied, the considerations of the implications prove the practical usefulness of integrated spatial indicators for modelling vulnerability to VBDs: they support decision makers to allocate resources for reducing existing susceptibilities and strengthening resilience in an effective manner to minimizing the burden of these diseases.

\section{References}

BlaschKe, T. (2006), The role of the spatial dimension within the framework of sustainable landscapes and natural capital. Landscape and Urban Planning, 75 (3-4), 198-226.

FeizizadeH, B., Jankowski, P. \& BlaschKe, T. (2014), A GIS based Spatially-explicit Sensitivity and Uncertainty Analysis Approach for Multi-Criteria Decision Analysis. Computers \& Geosciences, 64, 81-95. 
Fekete, A., DAmm, M. \& Birkmann, J. (2010), Scales as a challenge for vulnerability assessment. Natural Hazards, 55, 729-747.

Hagenlocher, M., Lang, S., Hölbling, D., Tiede, D. \& Kienberger, S. (2014), Modeling hotspots of climate change in the Sahel using object-based regionalization of multi-dimensional gridded datasets. IEEE Journal of Selected Topics in Applied Earth Observations and Remote Sensing, 7 (1), 229-234.

Hagenlocher, M., Delmelle, E., Casas, I. \& Kienberger, S. (2013), Assessing socioeconomic vulnerability to dengue fever in Cali, Colombia: statistical vs expertbased modeling. International Journal of Health Geographics, 12, 36.

IPCC (2014), Climate Change 2014: Impacts, Adaptation, and Vulnerability. Summary for Policy Makers. http://ipcc-wg2.gov/AR5/images/uploads/IPCC_WG2AR5_SPM_Approved.pdf.

Kienberger, S., Hagenlocher, M., Delmelle, E. \& CASas, I. (2013a), A WebGiS tool for visualizing and exploring socioeconomic vulnerability to dengue fever in Cali, Colombia. Geospatial Health, 8 (1), 313-316.

Kienberger, S., BLASCHKE, T. \& ZAIDI, R. Z. (2013b), A framework for spatio-temporal scales and concepts from different disciplines: the 'vulnerability cube'. Natural Hazards, 68, 1343-1369.

Lang, S., Kienberger, S., Tiede, D., Hagenlocher, M. \& Pernkopf, L. (2014), Geons domain-specific regionalization of space. Cartography and Geographic Information Science, 41 (03), 214-226.

Lang, S., Albrecht, F., Kienberger, S. \& Tiede, D. (2010), Object validity for Operational tasks in a policy context. Journal for Spatial Science, 55 (1), 9-22.

LIN, B. B. \& MoREFIELD, P. E. (2011), The vulnerability cube: a multi-dimensional framework for assessing relative vulnerability. Environmental Management, 48 (3), 631-643.

Magori, K. \& DraKe, J. M. (2013), The Population Dynamics of Vector-borne Diseases. Nature Education Knowledge, 4 (4), 14.

Openshaw, S. (1984), The modifiable areal unit problem. Norwich, Concepts and Techniques in Modern Geography.

Preston, B. L., Yuen, E. J. \& Westaway, R. M. (2010), Putting vulnerability to climate change on a map: a review of approaches, benefits and risks. Sustainability Science, online.

Simmons, C. P., Farrar, J. J., Nguyen, V. V. \& Wills, B. (2012), Dengue. The New England Journal of Medicine, 366 (15), 1423-32.

Stratton, L. M., O’Neill, S., Kruk, M. E. \& Bell, M. L. (2008), The Persistence of Malaria: Addressing the Fundamental Causes of a Global Killer. Social Science and Medicine, 67, 854-862.

WU, J. \& HARBIN, L. (2006), Concepts of scale and scaling. In: Wu, J., JONES, K. B., LI, H. \& LOUCKS, O. L. (Eds.), Scaling and uncertainty analysis in ecology: methods and applications. Springer, Berlin, 3-15.

WHO (2013), World Malaria Report 2013. http://apps.who.int/iris/bitstream/10665/97008/1/9789241564694_eng.pdf (January 15, 2014). 